\title{
ON FUNCTIONS WITH SUMMABLE APPROXIMATE PEANO DERIVATIVE
}

\author{
CHENG-MING LEE
}

\begin{abstract}
Let $\boldsymbol{n}$ be a positive integer and $\boldsymbol{F}$ a function defined on a closed interval $I$. For $x$ in $I$, let the $n$th approximate Peano derivative of $F$ at $x$, if it exists, be denoted as $F_{(n)}(x)$. For $n=1$, the existence of $F_{(n-1)}(x)$ will simply mean that the function $F_{(0)}(\equiv F)$ is approximately continuous at $x$. Then the following theorem is proved, noting that the phrase "for nearly all $x$ in $\Gamma$ " means "for all $x$ in $I$ except perhaps for those points in a countable subset of $\Gamma$ ", THeorem $\mathrm{A}_{n}$. Let $F_{(n-1)}(x)$ exist finitely for all $x$ in I. If $F_{(n)}(x)$ exists finitely for nearly all $x$ in I and is summable on $I$, then $F_{(n-1)}$ is absolutely continuous in $I$.
\end{abstract}

1. For $n=1$, Theorem $A_{n}$ is clearly a generalization of the well-known result (cf. Goffman [1], where a short transparent proof was given): A function whose ordinary derivative exists everywhere and is summable is absolutely continuous. The proof to be given is somehow rather involved. In fact, we need the following two lemmas, which seem to be interesting by themselves.

LEMMA 1. Let $F$ be approximately continuous in I and

$$
\lim \sup \operatorname{ap}|F(x+h)-F(x)| /|h|<+\infty
$$

for nearly all $x$ in $I$. Then $F$ is $[A C G]$ on I.

That $F$ is [ACG] on $I$ means that $I$ is the union of countably many closed sets on each of which the function $F$ is AC (i.e., absolutely continuous in the wide sense, see Saks [6]). Note that Saks' definition of ACG does not require the sets involved to be closed. However, his definition requires the function to be continuous. This ensures that a function ACG in Saks' sense is [ACG] since if a continuous function is $\mathrm{AC}$ on a set, it is $\mathrm{AC}$ on the closure of this set.

LEMMA 2. Let $H$ have the Darboux property and be $[A C G]$ on I. If $H_{(1)}(x)=0$ for almost all $x$ in $I$, then $H$ is constant on $I$.

Lemma 1 seems to be known for a long time (see Ridder [5]). We will give it a brief proof. Lemma 2 can be obtained from a theorem in [4], which gives an interesting characterization for monotone functions. However, we choose to give Lemma 2 a direct proof here.

We are unable to obtain a result similar to Lemma 1 for the proof of

Received by the editors July 2, 1975.

AMS (MOS) subject classifications (1970). Primary 26A15, 26A24; Secondary 26A48.

Key words and phrases. Approximate continuity, approximate Peano derivative, absolute continuity, generalized absolute continuity, monotone increasing, summable functions. 
Theorem $\mathrm{A}_{n}$ for $n \geqslant 2$. Instead, for the proof to be given for $n \geqslant 2$, we will base on the following two results. The first one, Theorem 1, is known (see Saks [6, p. 191]) and, in fact, its application in the study of AC property for everywhere differentiable functions is also well known (see, e.g. Rudin [7]). The second one, Theorem 2 , is recently due to the author [3].

THeOREM 1. Let $f$ be summable on 1 . Then for each $\varepsilon>0$ there exists a lower semicontinuous summable function $g$ such that $g(x)>-\infty$ and $g(x) \geqslant f(x)$ for all $x$ in $I$ and

$$
\int_{c}^{d} g(x) d x<\varepsilon+\int_{c}^{d} f(x) d x
$$

for all $c, d$ in $I$ with $c<d$.

THEOREM 2. Let $n \geqslant 2$ and suppose that $H_{(n-1)}(x)$ exists finitely for all $x$ in $I$. If $u H_{(n)}(x) \geqslant 0$ for almost all $x$ in $I$ and $u H_{(n)}(x) \geqslant-\infty$ for nearly all $x$ in $I$, or more generally, if $u_{0} H_{(n)}(x) \geqslant 0$ for almost all $x$ and $u_{0} H_{(n)}(x)>-\infty$ for nearly all $x$ in $I$, then $H_{(n-1)}$ is monotone increasing and continuous in $I$.

Here, as in [3] $u H_{(n)}(x)$ is the $n$th upper approximate Peano derivate of $H$ at $x$, while $u_{0} H_{(n)}(x)$ denotes the extended real number obtained by taking in the definition of $u H_{(n)}(x)$ the ordinary lim sup instead of the approximate lim sup. Later on in the proof of Theorem $A_{n}$, we will also use $l H_{(n)}(x)$ and $l_{0} H_{(n)}(x)$ to denote the corresponding lower values.

2. Proof of Lemma 1. For each positive integer $n$ and each $x$ in $I$, let $B(n, x)=\{y:|F(y)-F(x)|<n|y-x|\}$, and let $E_{n}$ be the set of all $x$ such that $|B(n, x) \cap J|>\frac{1}{2}|J|$ for all intervals $J \subset I$ with $x$ in $J$ and $|J|<1 / n$. Then as $F$ is approximately continuous in $I$, one shows that for any $x_{1}, x_{2}$ in the closure of $E_{n}$ with $\left|x_{2}-x_{1}\right|<1 / n$,

$$
\left|F\left(x_{2}\right)-F\left(x_{1}\right)\right| \leqslant n\left|x_{2}-x_{1}\right| \text {. }
$$

Hence, $F$ is AC on the closure of each $E_{n}$.

Now, let $E$ be the set of all points $x$ such that

$$
\lim \sup \operatorname{ap}|F(x+h)-F(x)| /|h|<+\infty \text {. }
$$

Then the set $S=I \sim E$ is at most countable, say $S=\left\{y_{1}, y_{2}, y_{3}, \ldots\right\}$, and denote $S_{j}=\left\{y_{j}\right\}$ for $j=1,2,3, \ldots, F$ is AC on each $S_{j}$ since it is a singleton. On the other hand, each $x$ in $E$ is a point in $E_{n}$ for some $n$. Hence, letting $C_{n}$ be the closure of $E_{n}$ for all $n$, one has $I=\left[\cup C_{n}\right] \cup\left[\cup S_{j}\right]$, a union of countably many closed sets on each of which $F$ is AC.

3. Proof of Lemma 2. Let $G$ be the set of all points $x$ in $I$ such that $H$ is constant on $J_{x} \cap I$ for some open interval $J_{x}$ containing $x$. Then $G$ is open in I. Suppose $G \neq I$. We will reach a contradiction. To see this, consider the nonempty closed set $E=I \sim G$. Since $H$ is [ACG] on $I$, it follows from the Baire category theorem that there exists $(c, d) \subset I$ such that $(c, d) \cap E \neq \varnothing$ and $H$ is $\mathrm{AC}$ on $[c, d] \cap E \equiv P$. Note that in each closed interval contained in $[c, d] \sim P$, the function $H$ is constant. Hence in the closure of each interval 
contiguous to $P$ (with respect to $[c, d]$ ), $H$ is constant since $H$ has the Darboux property. Then it follows easily that $H$, being AC on the closed set $P$, is AC on the whole interval $[c, d]$. Now, using this fact as well as that $H_{(1)}(x)=0$ for almost all $x$ in $[c, d]$, we show that $H$ is constant on $[c, d]$. It clearly suffices to show that $H(d)=H(c)$. To this end, let $\varepsilon>0$ be given but arbitrary, and let $A$ be the set of all $x$ in $[c, d]$ such that there exists a sequence of points $x_{i}$ with $x_{i} \neq x$ and $x_{i} \rightarrow x$ such that $\left|H\left(x_{i}\right)-H(x)\right|<\varepsilon\left|x_{i}-x\right|$. Then $|A|=d-c$ since $H_{(1)}(x)=0$ for almost all $x$ in $[c, d]$. Furthermore, the family of all intervals with $x$ and $x_{i}$ as the end points covers $A$ in the Vitali sense. Hence for each $\delta>0$, there exists a finite set $\left\{\left[y_{k}, y_{k}^{\prime}\right]\right\}$ of mutually exclusive intervals from the family such that $\sum\left(y_{k}^{\prime}-y_{k}\right) \geqslant(d-c)-\delta$. Then $\sum\left(t_{j}^{\prime}-t_{j}\right)<\delta$, where $\left\{\left(t_{j}, t_{j}^{\prime}\right)\right\}$ is the set of all subintervals of $[c, d]$ complementary to the set $\left\{\left[y_{k}, y_{k}^{\prime}\right]\right\}$. As $H$ is AC on $[c, d]$, choosing $\delta$ small enough, one has

$$
\sum\left|H\left(t_{j}^{\prime}\right)-H\left(t_{j}\right)\right|<\varepsilon .
$$

Hence

$$
\begin{aligned}
|H(d)-H(c)| & \leqslant \sum\left|H\left(y_{k}^{\prime}\right)-H\left(y_{k}\right)\right|+\sum\left|H\left(t_{j}^{\prime}\right)-H\left(t_{j}\right)\right| \\
& \leqslant \varepsilon \sum\left(y_{k}^{\prime}-y_{k}\right)+\varepsilon \leqslant \varepsilon[d-c+1] .
\end{aligned}
$$

As $\varepsilon$ is arbitrary, we conclude that $H(d)=H(c)$. Thus, we have proved that $H$ is constant on $[c, d]$, contradicting the fact that $(c, d) \cap E \neq \varnothing$. Hence we must have $G=I$. Then, using the Heine-Borel theorem, one sees easily that $H$ is constant on $I$, completing the proof.

4. Proof of Theorem $\mathrm{A}_{n}$. Let $K(x)=\int_{a}^{x} F_{(n)}(t) d t$ for all $x$ in $I$, where $a$ is the left end point of $I$. Then it is well known that $K$ is AC on $I$ and $K^{\prime}(x)=F_{(n)}(x)$ for almost all $x$ in $I$.

(i) For the case $n=1$, consider the function $H=K-F$. Clearly, $H$ is approximately continuous in $I$ and hence $H$ has the Darboux property in $I$. Furthermore, noting that $F$ is [ACG] on $I$ by Lemma 1 , one sees that $H$, as a difference of an AC and an [ACG] function, is [ACG] on $I$. It then follows from Lemma 2 that $H$ is constant on $I$ since $H_{(1)}(x)=K^{\prime}(x)-F_{(1)}(x)=0$ for almost all $x$ in $I$. Hence $F(=K-H)$ is AC on $I$.

(ii) For the cases $n \geqslant 2$, let $f=F_{(n)}$, and for each $\varepsilon>0$ let $g$ be as in Theorem 1. Then consider the function $H=G-F$, where $G$ is a function such that its $(n-1)$ th ordinary derivative $G^{(n-1)}(x)=M(x) \equiv \int_{a}^{x} g(t) d t$. Then $H_{(n-1)}=G^{(n-1)}-F_{(n-1)}=M-F_{(n-1)}$ on $I$. We will show further that $u_{0} H_{(n)}(x) \geqslant 0$ for nearly all $x$ in $I$ (see the remark following Theorem 2 for the notations involved). To this end, note first that $l_{0} M_{(1)}(x) \geqslant g(x)$ for all $x$ in $I$ since $g$ is lower semicontinuous in $I$. Furthermore, by a simple application of the following easy inequality (see Hobson [2])

$$
\lim \inf \frac{A(h)}{B(h)} \geqslant \lim \inf \frac{A^{\prime}(h)}{B^{\prime}(h)},
$$

one has $l_{0} G_{(n)}(x) \geqslant l_{0} M_{(1)}(x)$ for all $x$ in $I$. Hence 


$$
\begin{aligned}
u_{0} H_{(n)}(x) & \geqslant u H_{(n)}(x) \geqslant l G_{(n)(x)-u F_{(n)}}(x) \\
& \geqslant l_{0} G_{(n)}(x)-u F_{(n)}(x) \geqslant l_{0} M_{(1)}(x)-u F_{(n)}(x) \\
& \geqslant g(x)-u F_{(n)}(x)
\end{aligned}
$$

for all $x$ in $I$. But $u F_{(n)}(x)=F_{(n)} \neq \pm \infty$ for nearly all $x$ in $I$, so that one has that $u_{0} H_{(n)}(x) \geqslant g(x)-F_{(n)}(x) \geqslant 0$ for nearly all $x$ in $I$. Therefore, applying Theorem 2, one has that $H_{(n-1)}$ is monotone increasing in $I$. Hence

$$
F_{(n-1)}(d)-F_{(n-1)}(c) \leqslant M(d)-M(c)<\varepsilon+\int_{c}^{d} f(t) d t
$$

for all $c, d$ in $I$ with $c<d$. But since $\varepsilon$ is arbitrary, one concludes that

$$
F_{(n-1)}(d)-F_{(n-1)}(c) \leqslant \int_{c}^{d} f(t) d t=K(d)-K(c) .
$$

Replacing, in the above argument, $F$ by $-F$, one obtains the inverse of the above inequality. Hence one concludes that the above inequality is, in fact, an equality. Therefore, $F_{(n-1)}$ is $\mathrm{AC}$ on $I$ since $K$ is.

5. Further remarks and acknowledgement. It is clear that Lemma 1 and Theorem 2 are more general than what is required for the proof of Theorem $A_{n}$. If the full force of both results are used, a slight modification of the proof of Theorem $A_{n}$ yields the following improvement.

TheOREM $\mathrm{B}_{n}$. Let $F_{(n-1)}(x)$ exist finitely for all $x$ in $I$, and $F_{(n)}(x)$ exist finitely for almost all $x$ in $I$ and be summable on I. If $u F_{(n)}(x)<+\infty$ and $l F_{(n)}(x)$ $>-\infty$ for nearly all $x$ in $I$, then $F_{(n-1)}$ is absolutely continuous in $I$.

We remark that Bagby and Ziemer in a recent paper [8] have mentioned that C. J. Neugebauer has proved the following result: A function whose approximate derivative exists everywhere and is summable is absolutely continuous. This is slightly less general than Theorems $A_{0}$ or $B_{0}$ here. On the other hand, in their paper [8] concerning functions of several real variables, Bagby and Ziemer have also established, among others, a result containing Neugebauer's result. The comparison of the results here and some in [8] for the special case of functions of a single real variable seems worthwhile to make a further detailed study in another paper.

The author wishes to thank the referee, who, among others, suggested the improved version of Lemma 1 and drew the author's attention to references [7] and [8].

\section{REFERENCES}

1. C. Goffman, On functions with summable derivative, Amer. Math. Monthly 78 (1971), 874-875. MR 44 \#4156.

2. E. W. Hobson, The theory of functions of a real variable, 3rd ed., Cambridge, 1927, p. 359.

3. C. M. Lee, On the approximate Peano derivatives, J. London Math. Soc. (to appear).

4. C. M. Lee and D. W. Solomon, On monotone functions and Perron and Denjoy integrals, Department of Mathematics, University of Wisconsin-Milwaukee (unpublished).

5. J. Ridder, Ueber die genseitigen Beziehungen vershieder approximativ stetiger Denjoy-PerronIntegrale, Fund. Math. 22 (1934), 136-162. 
6. S. Saks, Theory of the integral, 2nd rev. ed., Monografie Mat., vol. VII, PWN, Warsaw, 1937.

7. W. Rudin, Real and complex analysis, 2nd ed., McGraw-Hill Ser. in Higher Math., McGraw-Hill, New York, 1974, pp. 179-180. MR 49 \#8783.

8. T. Bagby and W. P. Ziemer, Pointwise differentiability and absolute continuity, Trans. Amer. Math. Soc. 191 (1974), 129 -148. MR 49 \#9129.

Department of Mathematics, University of Wisconsin-Milwaukee, Milwaukee, WisconSIN 53201 